\title{
Situação sócio-econômica e condições de vida: comparação de duas coortes de base populacional no Sul do Brasil
}

\author{
Socioeconomic situation and living conditions: \\ comparison of two population-based cohorts from \\ southern Brazil
}

Elaine Tomasi 1

Fernando C. Barros 1

Cesar G. Victora 1 1 Departamento de Medicina
Social, Faculdade de
Medicina, Universidade
Federal de Pelotas. C. P. 464,
Pelotas, RS 96001-970, Brasil.

\begin{abstract}
Socioeconomic and family characteristics of two cohorts of babies born in 1982 and 1993 in Pelotas (Southern Brazil) were compared. There were 6,011 births in 1982 and 5,304 in 1993. In relation to family income, there were fewer poor babies in 1993; $60.8 \%$ of the families earned less than 3 times the monthly minimum wage in 1993, as compared to 69.5\% in 1982. Sanitary conditions also improved over the decade, and the proportion of families with running water and flush toilets increased by 10\%. On the other hand, there were no changes in the proportion of single-parent families or availability of home appliances like radios, stoves, and refrigerators. The mean number of persons per household increased from 3.0 in 1982 to 3.2 in 1993. In general, comparison of the two birth cohorts in this city suggests an improvement in quality of living over the time period for families with newborns. This finding should be taken into account when studying the evolution of health indicators over the course of the decade.
\end{abstract}

Key words Cohort Study; Socioeconomic Characteristics; Health Indicators

Resumo Com base em duas coortes de crianças nascidas na zona urbana de Pelotas, Rio Grande do Sul-6.011 em 1982 e 5.304 em 1993 -, foi possível comparar alguns indicadores sócio-econômicos e características da habitação de famílias que tiveram filhos nestes anos. Em 1993, nasceram menos crianças pobres: $60,8 \%$ das famílias tinham renda inferior a três salários mínimos por mês comparadas com 69,5\% em 1982. O saneamento melhorou bastante, subindo dez pontos percentuais na cobertura de água encanada e de sanitário com descarga. A inserção materna no trabalho remunerado cresceu de $34 \%$ para 38\%. Por outro lado, não houve alteração na proporção de famílias com a presença do pai e na proporção de famílias que dispunham de rádio, fogão e geladeira, embora estes dois itens tenham apresentado importantes variações conforme a renda familiar. O número médio de pessoas por dormitório aumentou de 3,0 em 1982 para 3,2 em 1993, destoando um pouco da tendencia positiva registrada nos demais indicadores. De modo geral, pode-se apontar para uma melhora nas condições de vida para quem teve filhos em Pelotas nesta última década, o que deve ser levado em conta quando da interpretação dos demais resultados dos estudos de coorte realizados.

Palavras-chave Estudos de Coorte; Condições Sócio-econômicas; Indicadores de Saúde 


\section{Introdução}

A determinação social nos indicadores de saúde infantil tem sido evidenciada em diversos estudos em todo o mundo (Townsend \& Davidson, 1982) e ficou clara em nosso meio principalmente com os resultados da pesquisa que acompanhou, desde o nascimento até os três anos de idade, uma coorte de crianças nascidas em Pelotas, RS, em 1982 (Victora et al., 1989). Com a nova coorte de 1993 , abriu-se a possibilidade de comparar alguns indicadores sócio-econômicos, selecionados para avaliar as condições de vida da população, na tentativa de subsidiar a discussão sobre a transição de saúde infantil nesta última década.

Não é intenção deste artigo avaliar as mudanças ocorridas no cenário econômico nacional na última década, mas há que se considerar o impacto causado por estas mudanças nas condições de vida das famílias que tiveram filhos em Pelotas nos anos estudados.

As principais características sócio-econômicas das crianças de 1982 influindo em seus perfis de morbimortalidade já foram descritas (Victora et al., 1989; Barros et al., 1984; Barros et al., 1987)

Assim, através de alguns indicadores selecionados, este artigo compara as condições de vida das famílias pelotenses entre 1982 e 1993.

\section{Metodologia}

As análises realizadas tomaram como variáveis independentes a renda familiar, expressa em salários mínimos mensais, e a classe social, composta a partir de informações sobre a renda, a escolaridade, a ocupação, a posição em relação aos meios de produção, o tipo de estabelecimento (para os trabalhadores por conta própria) e o número de empregados (para os empregadores) do chefe da família, conforme operacionalização proposta por Bronfman e colaboradores (Bronfman et al., 1988). A informação sobre a renda foi obtida na entrevista hospitalar, tanto em 1982 quanto em 1993. Já as informações necessárias sobre classe social para a primeira coorte foram obtidas em 1986 (durante o terceiro acompanhamento das crianças nascidas em 1982), e para a segunda coorte, na entrevista hospitalar.

As informações referentes ao saneamento básico, presença do pai, trabalho materno remunerado, posse de eletrodomésticos, pessoas por dormitório e número de moradores foram obtidas no primeiro acompanhamento para a coorte de 1982 (crianças com 15 meses em média), e na visita de 12 meses para a coorte de 1993, o que garante a sua comparabilidade.

O detalhamento dos métodos utilizados no estudo de 1982 foi objeto de um outro artigo prévio (Barros et al., 1990) e também está descrito nesta publicação (Victora et al., 1996).

\section{Resultados}

Dos 5.304 nascimentos ocorridos na zona urbana de Pelotas em 1993, dispõe-se de informação sobre a renda familiar para 5.190 crianças (98\%). A distribuição percentual destes nascimentos nos diferentes grupos de renda e sua comparação com os nascimentos de 1982 são mostrados na Figura 1.

Pode-se observar uma diminuição nas proporções dos grupos de até três salários mínimos mensais e um aumento nos grupos de renda mais alta. Estes dados indicam que uma menor proporção de mães de baixa renda tiveram filhos em 1993, se comparadas com as de maior poder aquisitivo.

Já em 1982, a classe social do chefe da família mostrou-se fortemente associada com a renda familiar (Victora et al., 1989). Em 1993, a associação permanece forte, observando-se alguns movimentos intraclasses distintos. A burguesia continua concentrada no grupo de mais alta renda. Na nova pequena burguesia e na pequena burguesia tradicional, há um deslocamento para os grupos de menor renda. O proletariado não típico também ficou mais pobre, com a proporção de renda inferior a um salário mínimo subindo de $7 \%$ para $12 \%$ nestes 11 anos. Por sua vez, o proletariado típico aumentou sua participação no grupo de renda de um a três salários e diminuiu no grupo inferior de renda familiar, restringindo-se as alterações a estes dois grupos nesta fração de classe. A situação do subproletariado, a exemplo da situação da burguesia, não apresentou alterações importantes.

A proporção de domicílios servidos de água potável subiu de $75 \%$ em 1982 para $85 \%$ em 1993. Ao analisarmos esta melhora por renda familiar, vemos que ela é sustentada pelo aumento expressivo da cobertura nos grupos de até três salários mensais. Nos demais grupos de renda, em que a cobertura já era bastante alta, estas proporções se mantiveram (Tabela 1). A mesma tendência praticamente se repete ao observarmos a sua distribuição conforme a classe social (Tabela 2).

Comportamento similar tiveram os dados referentes à presença de sanitário com descar- 
ga: de 75\% em 1982, passou para 84\% em 1993. Entre as famílias que ganhavam menos de um salário mínimo em 1982, apenas metade dispunha de sanitário com descarga em sua moradia. Em 1993, esta proporção era de $62 \%$. Outro aumento importante foi observado entre quem ganhava de um a três salários, indo de $71 \%$ em 1982 a 84\% em 1993 (Tabela 1). Entretanto, chama a atenção que o subproletariado apresente uma melhora muito pequena neste indicador (Tabela 2).

Praticamente não houve mudança na proporção de crianças que viviam em companhia de seu pai: 89\% em 1982 e 88\% em 1993. Entretanto, se observarmos no interior dos grupos de renda, veremos que, entre os mais pobres, a proporção de pais em casa aumentou de $78 \%$ para $88 \%$ nestes 11 anos, e em todos os demais grupos a proporção diminuiu (Tabela 1). Para os diferenciais de classe, a variável apresenta o mesmo comportamento (Tabela 2).

Na última década, subiu de $33 \%$ para $38 \%$ a proporção de mães que, por ocasião do primeiro ano de vida de seus filhos, exercia atividade remunerada fora do domicílio. Com exceção dos grupos de renda de até um salário e de seis
Figura 1

Distribuição das crianças conforme a renda familiar. Pelotas, RS, 1982-1993.

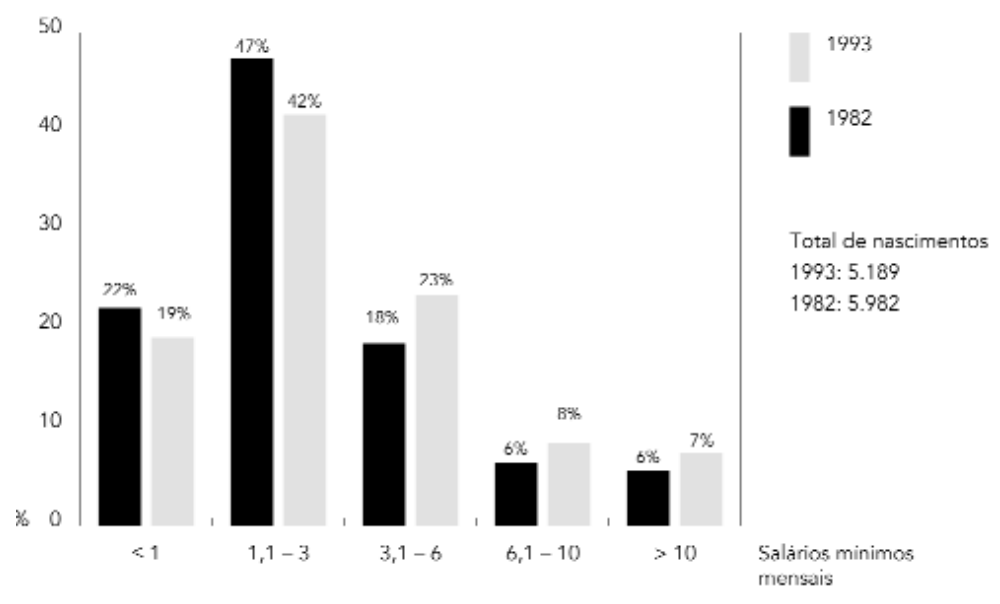

Tabela 1

Característica da habitação, presença do pai e trabalho materno conforme a renda familiar mensal.

Pelotas, RS, 1982-1993.

\begin{tabular}{|c|c|c|c|c|c|c|}
\hline & \multicolumn{6}{|c|}{ Grupos de Renda Familiar* } \\
\hline & $\leq 1$ & $1.1-3$ & $3.1-6$ & $6.1-10$ & $>10$ & Todos \\
\hline \multicolumn{7}{|c|}{ Água encanada (\%) } \\
\hline 1982 & 50 & 72 & 93 & 99 & 100 & 75 \\
\hline 1993 & 65 & 85 & 93 & 99 & 100 & 85 \\
\hline \multicolumn{7}{|c|}{ Sanitário com descarga (\%) } \\
\hline 1982 & 51 & 71 & 93 & 100 & 100 & 75 \\
\hline 1993 & 62 & 84 & 94 & 99 & 100 & 84 \\
\hline \multicolumn{7}{|c|}{ Pai vive com a criança (\%) } \\
\hline 1982 & 78 & 90 & 94 & 93 & 97 & 89 \\
\hline 1993 & 88 & 87 & 86 & 91 & 92 & 88 \\
\hline \multicolumn{7}{|c|}{ Trabalho materno (\%) } \\
\hline 1982 & 36 & 24 & 38 & 57 & 54 & 33 \\
\hline 1993 & 33 & 32 & 43 & 55 & 58 & 38 \\
\hline \multicolumn{7}{|c|}{ Possui rádio, TV e geladeira (\%) } \\
\hline 1982 & 37 & 66 & 90 & 98 & 100 & 69 \\
\hline 1993 & 37 & 66 & 83 & 93 & 100 & 69 \\
\hline \multicolumn{7}{|c|}{ Média de pessoas por dormitório } \\
\hline 1982 & 5,3 & 4,9 & 4,5 & 4,5 & 4,4 & 4,8 \\
\hline 1993 & 5,1 & 4,9 & 4,9 & 4,5 & 4,8 & 4,9 \\
\hline \multicolumn{7}{|c|}{ Total de Crianças } \\
\hline 1982 & 1321 & 2837 & 1105 & 383 & 336 & 5982 \\
\hline 1993 & 984 & 2165 & 1218 & 437 & 386 & 5190 \\
\hline
\end{tabular}

* Em 1982 e em 1993, informação de renda familiar em salários mínimos mensais. 
Características da habitação, presença do pai e trabalho materno conforme a classe social do chefe da família. Pelotas, RS, $1986-1993$.

\begin{tabular}{|c|c|c|c|c|c|c|c|}
\hline & \multicolumn{7}{|c|}{ Classe Social* } \\
\hline & Subploretariado & $\begin{array}{l}\text { Proletariado } \\
\text { Típico }\end{array}$ & $\begin{array}{l}\text { Proletariado } \\
\text { não típico }\end{array}$ & $\begin{array}{l}\text { Pequena } \\
\text { Burguesia } \\
\text { Tradicional }\end{array}$ & $\begin{array}{l}\text { Nova } \\
\text { Pequena } \\
\text { Burguesia }\end{array}$ & Burguesia & Todas \\
\hline \multicolumn{8}{|c|}{ Água encanada (\%) } \\
\hline 1986 & 44 & 62 & 82 & 87 & 100 & 100 & 76 \\
\hline 1993 & 68 & 79 & 90 & 94 & 100 & 100 & 87 \\
\hline \multicolumn{8}{|c|}{ Sanitário com descarga (\%) } \\
\hline 1986 & 55 & 59 & 81 & 87 & 100 & 100 & 75 \\
\hline 1993 & 57 & 78 & 90 & 93 & 100 & 100 & 85 \\
\hline \multicolumn{8}{|c|}{ Pai vive com a criança (\%) } \\
\hline 1986 & 73 & 88 & 91 & 94 & 95 & 93 & 90 \\
\hline 1993 & 80 & 92 & 89 & 88 & 91 & 100 & 89 \\
\hline \multicolumn{8}{|c|}{ Trabalho materno (\%) } \\
\hline 1986 & 41 & 27 & 36 & 31 & 47 & 27 & 34 \\
\hline 1993 & 42 & 33 & 35 & 42 & 78 & 43 & 38 \\
\hline \multicolumn{8}{|c|}{ Possui rádio, TV e geladeira (\%) } \\
\hline 1986 & 36 & 55 & 77 & 84 & 99 & 100 & 70 \\
\hline 1993 & 36 & 58 & 76 & 81 & 98 & 100 & 70 \\
\hline \multicolumn{8}{|c|}{ Média de pessoas por dormitório } \\
\hline 1986 & 3,8 & 3,5 & 2,9 & 2,7 & 1,9 & 1,9 & 3,0 \\
\hline 1993 & 3,9 & 3,4 & 3,2 & 3,0 & 2,3 & 1,8 & 3,2 \\
\hline \multicolumn{8}{|c|}{ Número médio de moradores } \\
\hline 1986 & 5,2 & 5,0 & 4,8 & 4,7 & 4,2 & 4,7 & 4,8 \\
\hline 1993 & 5,6 & 5,2 & 4,7 & 4,7 & 4,5 & 3,8 & 4,8 \\
\hline \multicolumn{8}{|c|}{ Total de crianças } \\
\hline 1986 & 468 & 1235 & 1973 & 647 & 287 & 58 & 4668 \\
\hline 1993 & 464 & 1260 & 2034 & 889 & 229 & 31 & 4907 \\
\hline
\end{tabular}

* Em 1986, informação do terceiro acompanhamento das crianças nascidas em 1982; em 1993, informação do Estudo Perinatal.

a dez salários, que diminuíram um pouco, nos demais houve um aumento da participação materna no trabalho remunerado. A maior diferença foi observada no grupo de um a três salários mínimos, no qual a proporção de mães trabalhando fora de casa subiu de 24\% em 1982 para $32 \%$ em 1993. Interessante observar também que no grupo de renda mais alta esta proporção variou de $54 \%$ para $58 \%$ no mesmo período (Tabela 1). Em relação à classe social, destacam-se os aumentos de 31 e 16 pontos percentuais para o trabalho materno remunerado na nova pequena burguesia e burguesia, respectivamente (Tabela 2 ).

Ao contrário das variáveis de saneamento básico, a posse dos três eletrodomésticos em funcionamento na moradia - aqui considerados rádio, televisão e geladeira - manteve-se inalterada no período estudado: pouco mais de dois terços em 1982 e em 1993. O exame deste indicador conforme renda familiar e classe social revela que esta situação também não teve mudanças conforme os grupos de renda e os diferenciais de classe.
Não houve mudanças na média do número total de pessoas na família no período estudado. O número médio de moradores teve um aumento importante no grupo que ganha de três a seis salários e entre os que ganham mais de dez salários mínimos mensais; nos demais, diminuiu um pouco ou permaneceu o mesmo. $\mathrm{O}$ proletariado não típico e a burguesia são as classes onde se observa uma diminuição no número de moradores entre 1986 e 1993 (Tabelas 1 e 2).

O número médio de pessoas por dormitório teve um discreto aumento de 3,0 em 1982 para 3,2 em 1993. Uma vez que o tamanho médio das famílias parece não ter aumentado neste período, pode-se atribuir esta mudança à também discreta diminuição do número médio de dormitórios na moradia - de 1,8 em 1982 para 1,6 em 1993.

Com exceção do grupo de renda de até um salário mínimo - que já apresentava e manteve a máxima aglomeração $(3,7$ pessoas por dormitório) -, em todos os demais grupos de renda observou-se um aumento destas médias. Me- 
recem destaque os grupos intermediários de renda, principalmente os de três a dez salários, nos quais o aumento chegou a cinco pontos percentuais (Tabela 1).

Excetuando-se o proletariado típico e a burguesia, em todas as outras classes consideradas houve um aumento na média de pessoas por dormitório, indicando uma pior condição de moradia para estas famílias (Tabela 2).

\section{Discussão}

A complexidade de uma dada situação sócioeconômica, com suas múltiplas determinações e variáveis, tem sido objeto de estudo de vários campos da ciência. A Epidemiologia, no âmbito de sua especificidade, tem feito contribuições importantes para o entendimento do processo saúde-doença, principalmente em seus determinantes sociais. Dentro destes limites, inscrevem-se as análises aqui realizadas, uma vez que dizem respeito a famílias pelotenses que tiveram filhos nos anos de 1982 e 1993, não sendo recomendadas quaisquer inferências dos resultados para a população geral da cidade, e tampouco para outras localidades brasileiras.

Por outro lado, para o sistema local de saúde, os resultados podem subsidiar o entendimento das modificações nos indicadores de

\section{Referências}

BARROS, F. C.; VICTORA, C. G.; GRANZOTO, J. A.; VAUGHAN, J. P. \& LEMOS JÚNIOR, A. V., 1984. Saúde perinatal em Pelotas, RS, Brasil. Fatores sociais e biológicos. Revista de Saúde Pública, 18:301-312.

BARROS, F. C.; VICTORA, C. G.; VAUGHAN, J. P. \& ESTANISLAU, H. J., 1987. Bajo peso al nacer en el municipio de Pelotas, Brasil: factores de riesgo. Boletín de la Oficina Sanitaria Panamericana, 102:541-553.

BARROS, F. C.; VICTORA, C. G. \& VAUGHAN, J. P., 1990. The Pelotas (Brazil) birth cohort study 1982-1987: strategies for following-up 6,000 children in a developing country. Paediatric and Perinatal Epidemiology, 4:267-282.

BRONFMAN, M.; LOMBARDI, C.; FACCHINI, L.; VICTORA, C. G.; BARROS, F. C.; BÉRIA, J. U. \& TEIXEIRA, A. M. B., 1988. Operacionalização do conceito de classe social em estudos epidemiológicos. Revista de Saúde Pública, 22:253-265.

TOWNSEND, P. \& DAVIDSON, N., 1982. Inequalities in Healht: The Black Report. Harmondsworth: Penguin Books Ltd. saúde ocorridas nesta última década, particularmente a queda dos coeficientes de mortalidade infantil e das incidências de eventos mórbidos entre as crianças menores de um ano.

Assim, comparando-se os indicadores sócio-econômicos disponíveis, percebe-se que houve uma melhora considerável nas condições de vida das famílias que tiveram filhos em 1982 e 1993. Esta melhora está apoiada principalmente pela melhor distribuição das famílias entre os grupos de renda, pelo crescimento do saneamento básico e pela maior inserção materna no trabalho remunerado. Apresentando situação inalterada no período, estão as variáveis relativas à presença do pai e à posse de eletrodomésticos. O único indicador que aponta para uma queda na qualidade de vida é a maior razão pessoas por dormitório. Registrou-se na cidade, neste período, a construção de grandes conjuntos habitacionais direcionados a famílias de baixa renda, conhecidos por suas reduzidas acomodações, o que talvez possa explicar esta maior aglomeração, já que o número médio de moradores não apresentou diferenças.

Os movimentos internos registrados entre a renda familiar e a classe social são importantes na medida em que indicam a existência de uma relação não necessariamente linear e simplista entre estes indicadores, mas podem expressar relações mais dinâmicas, a exemplo das relações sociais.

VICTORA, C. G.; BARROS, F. C.; HALPERN, R.; MENEZES, A. M.; HORTA, B. L.; TOMASI, E.; WEIDERPASS, E.; CESAR, J. A.; OLINTO, M. T.; GUIMARÃES, P. R. V.; GARCIA, M. M. \& VAUGHAN, J. P. Estudo longitudinal da população materno-infantil de Pelotas, localidade da Região Sul do Brasil, 1993: aspectos metodológicos e resultados preliminares. Revista de Saúde Pública, (no prelo).

VICTORA, C. G.; BARROS, F. C.; TOMASI, E.; MENEZES, A. M.; HORTA, B. L.; WEIDERPASS, E.; CESAR, J. A.; COSTA, J. S. D.; OLINTO, M. T.; HALPERN, R.; GARCIA, M. M. \& VAUGHAN, J. P., 1996. Tendências e diferenciais na saúde maternoinfantil: delineamento e metodologia das coortes de 1982 e 1993 de mães e crianças de Pelotas, RS. Cadernos de Saúde Pública, 12(supl. 1):7-14.

VICTORA, C. G.; BARROS, F. C. \& VAUGHAN J. P., 1989. Epidemiologia da Desigualdade. 2a ed., São Paulo: Hucitec. 